\title{
Evaluation of Practice of Prescription of the Corticotherapy in the Department of Internal Medicine of the University Hospital of Point G from 2009 to 2013
}

\author{
Abdel Kader Traoré1, Assétou Soukho Kaya', Djibril Sy¹, Djenebou Traoré1, \\ Alassane Doumbia1, Ilo Diall'2, Nangou Tolo1, Hadiza Amadou Kaïlou', Karim Dao', \\ Boua Daou Camara1, Ganda Soumaré1, Mamadou Dembélé1, Hamar Alassane Traoré1 \\ ${ }^{1}$ Department of Internal Medecine, University Hospital of Point G, Bamako, Mali \\ ${ }^{2}$ Department of Cardiology, University Hospital of Point G, Bamako, Mali \\ Email: traoreak@gmail.com
}

How to cite this paper: Traoré, A.K., Kaya, A.S., Sy, D., Traoré, D., Doumbia, A., Diall, I., Tolo, N., Kaïlou, H.A., Dao, K., Camara, B.D., Soumaré, G., Dembélé, M. and Traoré, H.A. (2019) Evaluation of Practice of Prescription of the Corticotherapy in the Department of Internal Medicine of the University Hospital of Point G from 2009 to 2013. Open Journal of Internal Medicine, 9, 89-96. https://doi.org/10.4236/ojim.2019.93013

Received: June 21, 2019

Accepted: September 3, 2019

Published: September 6, 2019

Copyright () 2019 by author(s) and Scientific Research Publishing Inc. This work is licensed under the Creative Commons Attribution International License (CC BY 4.0).

http://creativecommons.org/licenses/by/4.0/

(c) (7) Open Access

\begin{abstract}
Introduction: It is estimated that about $0.2 \%$ to $0.5 \%$ of French populations received a long-term systemic corticosteroid therapy. In Mali, from March, 2007 to February, 2008, in National Center for Disease Control and Prevention, $19.2 \%$ of hospitalized patients (24/125) received long-term corticosteroid therapy. Until now, the department of internal medicine of the university hospital of Point G hasn't done any research on the corticosteroid therapy specially. Therefore, we initiated this work to evaluate our practice of prescription of corticosteroid. The target of the study was to evaluate practical activity of prescription of corticosteroid of internal medicine. Methods: Based on all hospitalized patient materials of the department of internal medicine of Point G regional university central hospital during January 2009-December 2013, we initiated this retrospective and descriptive study. This study was based on all hospitalized patients receiving corticosteroid in the time of researching, without distinguishing their sex or age. Results: During the period of study, the department of internal medicine of point $\mathrm{G}$ regional central hospital had received 2155 patients; 64 were eligible: prevalence of $2.97 \%$. Most patients were female, accounting for $87.5 \%$ with a sex-ratio of 0.14 . The average age of our patients was $39.11 \pm 16.92$ years, ranging from 14 to 97 years. Physicians enrolled in the study Specialized Diploma (DES) and Internal represented $79.7 \%$ of prescribers. As pretreatment assessment, only 2 patients (3.12\%) had carried out all of the standard balance (NFS, electrolytes, calcium, blood glucose and serum protein). The most common glucocorti-
\end{abstract}


coide was prednisone. Solid tumors represented $31.25 \%$ (20 cases) of the diseases treated, followed by systemic lupus erythematosus with $15.62 \%$ (10 cases). In addition, there are two cases of adrenals insufficiency (3.1\%). In Point $\mathrm{G}$ internal medicine, pre-therapy evaluation and methods about corticosteroids therapy were insufficient and different.

\section{Keywords}

Pratiques, Corticothérapies, Médecine Interne, Bamako, Mali

\section{Introduction}

Corticosteroid is a derivative compounded by natural hormones, cortisol and prednisone, whose characters are a stronger anti-inflammatory power and, conversely, a lesser mineralocorticoid effect.

Statistically, there were $0.2 \%$ to $0.5 \%$ of general populations receiving long-term systemic corticosteroid therapy [1].

From January 1st, 1989-December 31st, 2008, an average 0.85\% of British population, received oral corticosteroid therapy [2].

In Mali, during March 2007-February 2008, in the National Center for Disease Control and Prevention $19.2 \%$ of hospitalized patients (24/125) received long-term corticosteroid therapy [3].

Since 1948 when glucocorticoid was firstly applied to therapy, it has been a top-ranking method of anti-inflammation therapy. The anti-inflammation power of its derivative (corticosteroid) for therapy was stronger than cortisol [4]. In the treatment of quantities of diseases, compounded glucocorticoid had a stronger power of anti-inflammation and immunosuppression at certain pharmacological doses. But it was necessary to add compounded glucocorticoid beyond physical dosage for above effects, and there were quantities of side-effect. Consequently, the cost of therapy cannot be neglected. Glucocorticoid has many effects for water-electrolyte balance as well as the metabolization of carbohydrates, proteins, and lipid. Besides, it must be considered while prescribing that treatments using glucocorticoid depress functions of the hypothalamic-pituitary-adrenal axis or the corticotropic axis [5]. Glucocorticoid (GC) had a quantitative and qualitative influence on the immune system and could destroy cellular immunity, but exerted almost no influence on humoral immunity. At the same time, there was a harmful influence on actors with inflammatory reactions [6].

Dosage and duration of treatment had a certain influence on reactions of hypothalamus-pituitary and the infection risk of corticosteroid treatments and the individual factors which are difficult to determine at present were also pretty important. Now it is difficult to define the least therapeutic dosage or the minimum therapeutic time to avoid its side-effect [5] [6].

So it was clear that corticosteroid treatments must obey a certain number of 
rules to avoid accidents and incidents. It was not rare that patients receiving systemic corticosteroid treatments also received supplements of vitamins, calcium and potassium, gastric protection and biphosphonate treatments. Doctors would provide the patients with advices nutrition and health protection, such as changing into the diet of low-sodium, low-carbogydrate and rich-protein as well as increasing regular physical exercise. Among above suggestions, it was rational to prevent osteoporosis by supplements of calcium and potassium, which belonged to recommendations agreed by two sides while other medication and methods of the diet were not recommendations [1] suggested.

At present, the department of internal medicine of Point G regional central hospital has not researched corticosteroid treatments specially. So what about our current practice? Therefore, we initiated this work to evaluate our practice of prescription of corticosteroid used by patients.

The target of this evaluation was to define Point G regional central hospital's frequency of prescription of corticosteroid, to define different treatment methods of using corticosteroid on the department of internal medicine and related side-effect, and to analyze assistant measures related to corticosteroid.

\section{Methods}

We made a descriptive study with a retrospective screening of our database concerning 64 hospitalized patients' in to the department of internal medicine of the Universitary Hospital of Point G in Bamako-Mali, from January 2008 to December 2013.

The inclusion of the patients have been was exhaustive, and concerned all the patients receiving corticosteroid treatment.

For all of them, we collect:

- The socio-demographics data (age, sex);

- The biologic data before and after corticosteroid treatment (Blood glucose, serum potassium, serum calcium, blood fat, polynuclear rate and rate of lymphocyte);

- Information about treatment (duration and posology of the corticoid treatment, supplement of potassium, calcium and vitamin D, Gastric protectionpronton-pomp inhibitors);

- The side-effects during corticosteroid treatment (acute adrenal insufficiency and rebound phenomenon);

- The diet with low sodium (control of carbohydrate and lipid, diet with rich protein);

- The regular exercise;

- The evolution of the treatment;

- Related data gaining and analysis was done using Epi info software.

\section{Results}

From January 2009-December 2013, the department of internal medicine of point $\mathrm{G}$ regional central hospital received 2155 patients totally, and 64 have been 
include to the, with a prevalence of $2.9 \%$.

The average age of patients was $39.11 \pm 16.92$ ranging from 14 to 97 . The women represent $87.5 \%$ with a sex/ratio of 0.14 . Physicians enrolled in the study was Specialized Diploma (DES) and Internal represented $79.7 \%$ of prescribers (Table 1).

As pretreatment assessment, only 2 patients (3.12\%) had carried out all of the standard balance (NFS, electrolytes, calcium, blood glucose and serum protein). In the department of internal medicine of Point G, pre-therapy evaluation and methods about corticosteroids therapy were insufficient and not standardized (Table 2).

Among the 64 cases included, for 36 patients prednisone have been used; for $56.25 \%$ of them, 25 patients received methylprednisolone (39.05\%) (Table 2).

Solid tumors accounted for $31.25 \%$ of treated pathologies. These solid tumors was rectal adenocarcinoma, bronchopulmonary cancer, breast cancer, ovarian cancer, pleural carcinoma, uterine leiomyosarcoma, osteogenic osteosarcoma, brain tumor, bladder tumor, gastric tumor, inguinal tumor and scrotal tumor. The systemic lupus erythematosus represented $15.62 \%(\mathrm{n}=10)$. Two cases of adrenal insufficiency have been observed (3.1\%) (Table 3).

Regarding other related methods, 52 patients were asked to have a diet with low sodium (81.25\%), 52 patients to supply potassium (81.25\%) and 31 patients

Table 1. Sociodemographic distribution of patients and the prescribers of corticotherapy $(\mathrm{n}=64)$.

\begin{tabular}{|c|c|c|}
\hline Distribution & Frequency & Percentage \\
\hline \multicolumn{3}{|l|}{ Rate of Age (Years Old) } \\
\hline $10-35$ & 14 & $21.88 \%$ \\
\hline $36-60$ & 30 & $46.87 \%$ \\
\hline $60-85$ & 16 & $25.00 \%$ \\
\hline$>85$ & 4 & $6.25 \%$ \\
\hline Average of Age & \multicolumn{2}{|c|}{$39.11 \pm 16.92$} \\
\hline Limit of Age & \multicolumn{2}{|c|}{14 to 97} \\
\hline \multicolumn{3}{|l|}{ Sex } \\
\hline Male & 30 & $46.88 \%$ \\
\hline Female & 34 & $54.12 \%$ \\
\hline Sex/Ratio & \multicolumn{2}{|c|}{0.14} \\
\hline \multicolumn{3}{|l|}{ Prescriber } \\
\hline DES or Resident & 51 & $79.7 \%$ \\
\hline Rheumatologist & 9 & $14.1 \%$ \\
\hline Hematologist & 1 & $1.6 \%$ \\
\hline Doctoral Candidate & 1 & $1.6 \%$ \\
\hline Internist physician & 1 & $1.6 \%$ \\
\hline Neurologist & 1 & $1.6 \%$ \\
\hline
\end{tabular}


Table 2. Distribution of patients according to the biological pre-treatment assessment, the corticosteroid used and by year of hospitalization.

\begin{tabular}{|c|c|c|}
\hline Biological pre-treatment assessment & Frequency & Percentage \\
\hline NFS & 44 & $48.43 \%$ \\
\hline Serum Calcium & 18 & $34.37 \%$ \\
\hline Ionogram & 18 & $32.81 \%$ \\
\hline GLU & 39 & $28.12 \%$ \\
\hline NFS + Ionogram + Serum Calcium + GLU + Protidemia & 2 & $10.93 \%$ \\
\hline IIPID BALANCE & 1 & $10.93 \%$ \\
\hline NFS + Ionogram + Serum Calcium & 2 & $53.12 \%$ \\
\hline NFS + Ionogram + Serum Calcium + GLU & 5 & $6.25 \%$ \\
\hline All the Biological pre-treatment assessment & & $3.12 \%$ \\
\hline \multicolumn{3}{|l|}{ Corticide Used } \\
\hline Prednisone & 29 & $45.30 \%$ \\
\hline Methylprednisolone & 19 & $29.70 \%$ \\
\hline Dexamethasone & 7 & $10.9 \%$ \\
\hline Betamethasone & 1 & $1.6 \%$ \\
\hline Dexamethasone and Prednisone & 1 & $1.6 \%$ \\
\hline Methyl Prednisolone then Prednisone & 5 & $1.6 \%$ \\
\hline Prednisolone & 1 & $1.6 \%$ \\
\hline Prednisone then Methylprednisolone & 1 & $1.6 \%$ \\
\hline \multicolumn{3}{|l|}{ Year of Hospitalization } \\
\hline 2013 & 23 & $35.90 \%$ \\
\hline 2012 & 13 & $20.30 \%$ \\
\hline 2011 & 10 & $15.60 \%$ \\
\hline 2010 & 9 & $14.10 \%$ \\
\hline 2009 & 9 & $14.10 \%$ \\
\hline
\end{tabular}

to supply vitamin and calcium (48.43\%). The prescription didn't ask low carbohydrate, a diet with rich-protein or regular exercise in the range of study. The time of corticosteroid treatment of 36 patients was over 15 days (56.25\%). Only one case used hydrocortisone (Table 4).

\section{Discussion}

Because this investigation was retrospective, it was difficult to be explained or contrasted with other rare cases in this domain. Regarding international bibliographies, we have seen less than 15 studies about the general prescription of corticotherapy.

In our study, the frequency of prescription of corticosteroid was $2.9 \%$. Petersen found that the frequency in the UK was $0.85 \%$ [2]. Fardet estimated that $0.2 \%$ to $0.5 \%$ of French populations used prescriptions of corticosteroid [1]. The difference could be caused by quantities of samples (the study of Petersen was 
Table 3. Distribution of patients according to the pathology treated.

\begin{tabular}{ccc}
\hline Pathology Treated & Frequency & Percentage \\
\hline Solid Tumors & 20 & $31.25 \%$ \\
Systemic Lupus Erythematosus & 10 & $15.62 \%$ \\
Non-Hodgkinian Lymphoma & 7 & $10.94 \%$ \\
Nephrotic Syndrome & 6 & $9.37 \%$ \\
Meningitis & 5 & $7.81 \%$ \\
Rheumatoid Arthritis & 2 & $3.12 \%$ \\
Pneumocystis & 2 & $3.12 \%$ \\
Systemic Immune Reconstitution Syndrome & 2 & $3.12 \%$ \\
Compressive Dyspneizing Polyadenopathy & 1 & $1.56 \%$ \\
Hodgkin's Disease & 1 & $1.56 \%$ \\
Acute Root Polyneuritis & 1 & $1.56 \%$ \\
Purpura Thrombopenic Idiopathic & 1 & $1.56 \%$ \\
Meningoencephalitis & 1 & $1.56 \%$ \\
Sharp Syndrome & 1 & $1.56 \%$ \\
Cerebral Abscess & 1 & $1.56 \%$ \\
Myelodysplasia & 1 & $1.56 \%$ \\
Medullary Infarction & 1 & $1.56 \%$ \\
Megaloblastic Anemia & 64 & $1.56 \%$ \\
Total & $100.0 \%$ \\
\hline Retal & 1 & \\
\hline & 1 & 1.50
\end{tabular}

Solid Tumors = Rectal adenocarcinoma, bronchopulmonary cancer, breast cancer, ovarian cancer, pleural carcinoma, uterine leiomyosarcoma, osteogenic osteosarcoma, brain tumor, bladder tumor, gastric tumor, inguinal tumor, scrotal tumor.

based on 4,518,753 patients).

The average age of patients was 39.11 years old from 14 to 97 years old. the median ages of short-term and long-term corticosteroid treatments were 56.1 and 67.4 years. In the study of Petersen [2], the difference also might be caused by our quantities of samples. In this study, the ratio of females was 0.88 , while in the study of Petersen [2] the ratio of males was 1.5. Prednisone was the most glucocorticoid of prescriptions in our study, accounting for $56.25 \%$ of total cases. In the study of Petersen [2], prednisolone represented $89.9 \%$ of total prescriptions, followed by dexamethasone, $5.1 \%$. This difference might be due to most of the patients in our study receiving treatment of solid tumors, followed by the treatment of systemic lupus erythematosus, while subjects of Petersen's [2] study mainly received treatment of pneumonopathy. In the study of Fardet [1], the incidence of nodular vasculitis and connective tissue was highest.

In this study, $81.25 \%$ of cases were suggested to have a low-salt diet, while $80 \%$ of cases were suggested so in the study of Fardet [1].

The lower carbohydrate, a diet with rich-protein or regular exercise were not asked in prescriptions in the range of this study.

In the study of Fardet, $40 \%$ of physicians wrote prescriptions of a low-sugar 
Table 4. Distribution of patients according to related assistant measures.

\begin{tabular}{lcc}
\hline \multicolumn{1}{c}{ Related measures } & Frequency & Percentage \\
\hline Low-sodium diet & 52 & $81.25 \%$ \\
Potassium supplementation & 52 & $81.25 \%$ \\
Vitamin and Calcium supplementation & 31 & $48.43 \%$ \\
Deworming & 21 & $32.81 \%$ \\
Gastric protection & 18 & $28.12 \%$ \\
Low-sodium diet + Potassium supplementation + & 7 & $10.93 \%$ \\
Vitamin-calcium supplementation + Deworming & & \\
Low-sodium diet + Potassium supplementation + & & $10.93 \%$ \\
Vitamin-calcium supplementation + Deworming + & 7 & \\
Gastric protection & & $6.25 \%$ \\
Low-sodium diet + Potassium supplementation + & & \\
Vitamin-calcium supplementation + Gastric protection & 4 & $0 \%$ \\
Carbohydrate controlled diet & & 0 \\
Protein-rich foods & 0 & 0 \\
Regular physical exercise & 0 &
\end{tabular}

diet usually; $20 \%$ of physicians suggested having a diet with rich protein, while less than $40 \%$ of physicians often suggested exercising regularly for $80 \%$ of patients.

$45.31 \%$ of patients in our studying cases received prescriptions of gastric protection. In the study of Fardet, 10\% of doctors usually wrote prescriptions of gastric protection for their patients. There were $81.25 \%$ of patients having prescriptions of potassium supplements in our studying cases. In the study of Fardet, $39 \%$ of doctors often wrote prescriptions of potassium supplements for their patients.

\section{Conclusion}

Recently the frequency of prescriptions of corticosteroid increased year by year. Related assistant measures and pre-treatment evaluation were largely different and insufficient. It needed cooperation with other researches and common suggestions of two sides to deal with this problem.

\section{Conflicts of Interest}

The authors declare no conflicts of interest regarding the publication of this paper.

\section{References}

[1] Fardet, L., Hanslik, T., Blanchon, T., Perdoncini-Roux, A., Kettaneh, A., Tiev, K.P., Turbelin, C., Dorleans, Y., Cabane, J., et al. (2008) Adjuvant Measures to Long-Term Systemic Corticosteroid Therapy: Practice Description of French Physicians. La Revue de Médecine Interne, 29, 975-980. 
https://doi.org/10.1016/j.revmed.2008.04.001

[2] Fardet, L., Petersen, I. and Nazareth, I. (2011) Description of Prescriptions for Oral Corticosteroids in the General Population. La Revue de Médecine Interne, 32, 594-599. https://doi.org/10.1016/j.revmed.2011.02.022

[3] Keita, A.C.F. (2011) Evaluation of the Cost of Long-Term General Corticosteroids in the Dermatology Department of the National Center for Disease Control and Prevention (CNAM). Essay, Med, Bamako, No. 11-M-303.

[4] Larbre, J.P. and Llorca, G. (1999) Corticosteroids: Use Principles and Rules. La Revue du Praticien, 49, 893-900.

[5] Bertherat, J. (2002) Corticotherapy and Adrenal Function. Ency Med Chir Nutrition 10-015-A-20, 6 p.

[6] Dussauze, H., Bourgault, I., Doleris, L.-M., Prinseau, J., Baglin, A. and Hanslik, T. (2007) Systemic Corticosteroids and Infectious Risk. La Revue de Médecine Interne, 28, 841-851. https://doi.org/10.1016/j.revmed.2007.05.030 